Published in final edited form as:

Obes Rev. 2016 December ; 17(12): 1245-1257. doi:10.1111/obr.12476.

\title{
Nutritional Interventions or Exposures in Infants and Children Aged up to Three Years and their Effects on Subsequent Risk of Overweight, Obesity, and Body Fat: a Systematic Review of Systematic Reviews
}

\author{
Bernadeta Patro-Gołąb, MD\#1, Bartłomiej M Zalewski, MD\#1, Maciej Kołodziej, MD1', Stefanie \\ Kouwenhoven, Bsc ${ }^{2}$, Lucilla Poston, PhD $^{3}$, Keith M Godfrey, PhD $^{4}$, Berthold Koletzko, MD ${ }^{5}$, \\ Johannes Bernard van Goudoever, MD ${ }^{2,6}$, and Hania Szajewska, MD1
}

${ }^{1}$ Department of Paediatrics, Medical University of Warsaw, Poland ${ }^{2} \mathrm{VU}$ University Medical Center Amsterdam, Department of Pediatrics, Amsterdam, The Netherlands ${ }^{3} \mathrm{KCL}$ Division of Women's Health, Women's Health Academic Centre, King's College London and Kings Health Partners, UK ${ }^{4}$ MRC Lifecourse Epidemiology Unit and NIHR Southampton Biomedical Research Centre, University of Southampton and University Hospital Southampton NHS Foundation Trust, UK ${ }^{5}$ Ludwig-Maximilians-Universität München, Division of Metabolic and Nutritional Medicine, Dr. von Hauner Children's Hospital, University of Munich Medical Centre, München, Germany ${ }^{6}$ Department of Paediatrics, Emma Children's Hospital, Amsterdam Medical Center, Amsterdam, The Netherlands

\# These authors contributed equally to this work.

Correspondence to: Bernadeta-Patro Gołąb, Medical University of Warsaw, Department of Paediatrics, Żwirki i Wigury 63A, 02-091 Warsaw, Poland, abpatro@yahoo.com.

Declaration of potential conflicts of interest

BPG received a research training fellowship grant from Nestle Nutrition Institute.

BMZ, MK declare no conflict of interest.

SK A part of her $\mathrm{PhD}$ thesis is to assess the effects of an infant formula with an optimized amino acid composition and a lower protein content on growth and body composition.

KMG received reimbursement for speaking at conferences sponsored by companies selling nutritional products and is part of an academic consortium that has received research funding from Abbott Nutrition, Nestec, and Danone.

The work of BK is financially supported by the Commission of the European Communities, the 7th Framework Programme Early Nutrition (FP7-289346), the Horizon 2020 research and innovation programme DYNAHEALTH (No 633595), and the European Research Council Advanced Grant META-GROWTH (ERC-2012-AdG - no.322605). Additional support is from the German Ministry of Education and Research, Berlin (Grant Nr. 01 GI 0825), the German Research Council (DFG), and the University of Munich Innovative Research Priority Project MC-Health. BK is a member of the National Breastfeeding Committee and tends to be biased towards breastfeeding. The Ludwig-Maximilians-Universität München, Germany and its employee, BK, have received support for scientific and educational activities by different nutritional companies, predominantly as part of publically funded research projects with support of the European Commission or German governmental research support.

JBvG is founder and director of the Dutch Human Donor Milk Bank and is a member of the Dutch Health Council. He holds patents on amino acid composition of infant formula.

HS has participated as a clinical investigator and/or speaker for companies selling nutritional products (Arla, Danone, HiPP, Nestle', Nestle' Mead Johnson).

LP has received support for scientific and educational activities from companies including Abbott Nutrition, Danone, Nestle, and Tate and Lyle, predominantly as part of publically funded research projects with support of the European Commission.

Contributors

All authors contributed to formulating the research question and designing the study. The first draft of the protocol was prepared by BMZ and BPG and further discussed by all authors. BPG, BMZ, MK, and SK searched specified data sources, selected articles, and extracted all necessary data. BPG and BMZ analyzed the data. BPG and BMZ wrote the first draft of the manuscript. All authors revised and/or commented on drafts. All authors accepted the final version of the manuscript. 


\section{Abstract}

This study performed as part of the international EarlyNutrition research project (www.projectearlynutrition.eu) provides a systematic review of systematic reviews on the effects of nutritional interventions or exposures in children (up to three years of age) on the subsequent risk of obesity, overweight, and adiposity. Electronic databases (including MEDLINE, EMBASE, and Cochrane Library) were searched up until September 2015. Forty systematic reviews were included. A consistent association of breastfeeding with a modest reduction in the risk of later overweight and obesity in childhood and adulthood was found (the odds decreased by $13 \%$ based on high quality studies), but residual confounding cannot be excluded. Lowering the protein content of infant formula is a promising intervention to reduce the risk of later overweight and obesity in children. There is no consistent evidence of an association of the age of introducing complementary foods, sugar-sweetened beverage, or energy intake in early childhood, with later overweight/obesity, but there are some indications of an association of protein intake during the complementary feeding period with later overweight/obesity. There was inadequate evidence to determine effects of other nutritional interventions or exposures, including modifications of infant formula composition, fat intake, or consumption of different food groups.

\section{Keywords}

obesity; systematic review; nutrition

\section{Introduction}

Early life exposures to suboptimal nutrition have been widely implicated in causal pathways leading to increased risk of disease in later life. 1 This concept has become known as 'early metabolic programming' or 'developmental programming' and was derived by evidence from animal studies 2 and later human epidemiological studies associating low birth weight with adverse cardiovascular and metabolic outcomes in adulthood.3,4 One of the hypotheses focuses on postnatal accelerated weight gain, suggesting that rapid weight gain in infancy can lead to increased risk of later obesity and related disorders.5,6,7 For example, higher protein intake in infancy has been reported to stimulate the secretion of insulin and insulinlike growth factor I and, thereby, increase weight gain and further obesity risk.8 In contrast, there is evidence suggesting that breastfeeding has a protective effect against overweight and obesity. 9

Many observational studies have examined the relationship between nutrition in infancy and early childhood and growth and child health outcomes, whereas intervention studies that can directly address causality are limited in number. A consequence of the paucity of intervention studies conducted in humans is that observational data are increasingly contributing to guideline development for dietary advice in infancy and early childhood, 10 a practice widely accepted to have considerable limitations. Systematic reviews have failed to improve clarity because of widely varying approaches. Moreover, guidelines on nutrition for infants and young children often fail to address longer-term outcomes, including the risk of cardiovascular disease, hypertension, overweight, obesity, and diabetes. There is also inconsistency amongst these recommendations for some health outcomes.11 This prompted 
the EarlyNutrition Project (www.project-earlynutrition.eu) to undertake a systematic review of published systematic reviews on the effects of nutritional interventions or exposures in children (up to three years of age) on the subsequent risk of obesity, overweight, and adiposity in order to develop new, improved strategies and recommendations on nutrition in this population.

This review was initiated as part of the development of recommendations by the EarlyNutrition Project on nutrition during pregnancy, lactation, and early childhood and consequences for offspring health and risk of later obesity.

\section{Methods}

\section{Criteria for considering studies for this review}

Types of studies-We included systematic reviews (of randomized controlled trials [RCTs] and/or observational studies) with/or without a meta-analysis. Systematic reviews of systematic reviews were also eligible for inclusion. We considered a review as 'systematic' if it was indicated to be by the authors and/or it had key characteristics of a systematic review, following the Cochrane Handbook:12 'a clearly stated set of objectives with predefined eligibility criteria for studies; an explicit, reproducible methodology; a systematic search that attempts to identify all studies that would meet the eligibility criteria; an assessment of the validity of the findings of the included studies, for example through the assessment of risk of bias; and a systematic presentation, and synthesis, of the characteristics and findings of the included studies. 'Non-systematic reviews and reviews of guidelines were excluded.

Types of participants/populations-Reviews must have included infants and young children (aged up to three years) representing the general population (including those whose mothers and/or fathers are overweight/obese). Systematic reviews of studies that recruited infants and young children with a wider range of age also were eligible for inclusion; however, we must have been able to extract data on children up to three years of age. We excluded systematic reviews that focused on studies conducted (i) in infants and children with specific disorders, (ii) in preterm infants or in SGA (small for gestational age) infants, and (iii) in children from mothers/fathers with specific disorders or conditions (mental or physical) other than overweight or obesity.

Types of interventions/ exposures-Reviews that assessed the effects of specific nutritional interventions/exposures in children were included. Behavior change interventions focused on improving nutrition were not a subject of this review. Similarly, mixed interventions/exposures (such as combinations of nutritional interventions/exposures together with physical activity) were not addressed in this review.

Type of comparator(s)/ controls-Usual standard care or diet; no intervention; various levels of exposure.

Type of outcome(s)—Our pre-specified primary outcomes measured at any age after birth included the following: obesity and overweight, as defined by the authors; body mass 
index (BMI) or changes in this parameter at different time intervals (as defined by the authors); fat mass; body fat percentage; and fat-mass index (measured with the use of different methods/devices). We excluded outcome measures focused on the presence of specific disorders such as asthma, epilepsy, etc. We chose the outcome assessment to be at any age after birth for few reasons: (i) the lack of a clearly defined cut-off time point to indicate the optimal time for assessment of the phenotypic effect of programming either in relation to the interval following the exposure or the age of the offspring; (ii) reviews often gather evidence on both immediate and longer-term effects of nutritional exposures; (iii) it is of interest to identify whether or not immediate and longer-term effects are in the same direction; differences in the direction of an effect could imply that mechanisms are dissimilar.

\section{Search strategy}

The Centre for Reviews and Dissemination (CRD, University of York) was involved in the development of our search strategy. Several electronic databases were searched for eligible systematic reviews, up until September 2015: Cochrane Database of Systematic Reviews (CDSR); The Database of Abstracts of Reviews of Effects (DARE); Ovid MEDLINE; and EMBASE (Biomedical and pharmacological bibliographic database). The reference lists from identified reviews were searched. Attempts to obtain reviews from other sources were made by a references' hand search of the included reviews, as well as by contact with experts in the field. The detailed search strategy for Ovid MEDLINE is shown in Table S1. Four reviewers (BMZ, MK, BPG, and SK) independently carried out the literature search. No language restrictions were applied. Systematic reviews meeting our inclusion criteria that had been published after September 2015 were also eligible, if only we were aware of such publications. However, no formal search was undertaken after September 2015.

\section{Selection process}

For this review, at least two of our four reviewers (BPG, BMZ, MK, SK) independently screened the titles and abstracts. Full texts of potentially eligible systematic reviews were further evaluated. If an update of a particular review was published, we included the most recent publication. Disagreements concerning the eligibility for inclusion were resolved by discussion until a consensus was reached. All reviewers agreed on the final set of included reviews.

\section{Data extraction}

For each included review, three reviewers (BPG, BMZ, MK) performed data extraction using a standardized table. Data extracted included first author, search strategy timeframe, number of studies included in total and involving children aged up to three years, age of participants, study design, intervention/exposure, primary outcomes, results, evidence quality, and comments.

\section{Data synthesis}

A narrative synthesis was undertaken. We did not intend to and did not perform a metaanalysis of the meta-analyses of included reviews due to the following reasons: (i) expected 
heterogeneity of included systematic reviews resulting mainly from a broadly defined clinical question; (ii) difficulty in avoiding the use of data from individual studies more than once and, therefore, inadequately increasing the statistical power when pooling data together.13 Moreover, whenever possible, we aimed to base our conclusions on the most recent and robust reviews, trying to avoid overestimating the effect due to duplication of studies.

\section{Quality assessment of the reviews}

Two reviewers independently assessed the methodological quality of each included review using the 'Assessment of Multiple Systematic Reviews' (AMSTAR) tool.14 To our knowledge, the AMSTAR tool is the only validated tool to assess the methodological quality of systematic reviews.13 The tool contains 11 questions with regard to the quality of the review. For the purposes of this document, subjectively, we assumed that scores of zero to four indicate a low quality review, five to eight, a moderate quality review, and, nine to 11 , a high quality review.

Formally, we did not assess the quality of identified systematic reviews of systematic reviews due to incompatibility of this kind of review with the AMSTAR tool.

\section{Risk of bias in included studies}

If originally assessed by the authors of the included reviews, we extracted data with respect to the risk of bias (methodological quality) of studies included in every eligible review. However, we neither performed a formal judgment of this assessment nor assessed individual studies ourselves. When formulating our conclusions, we took into account both the quality of the review and the overall quality of evidence that additionally resulted from the quality of the included studies.

\section{Results/Effects of Interventions/Exposures}

\section{Breastfeeding}

Nine systematic reviews $9,15,16,17,18,19,20,21,22$ and two systematic reviews of systematic reviews 23,24 have evaluated the association of breastfeeding (BF) with later overweight and obesity (Table S2).

Included reviews were heterogeneous in terms of methodology, clinical aspects, and methodological quality. Only one (a Cochrane review) was of high quality; 18 most were classified as being of moderate quality, and one, of low quality.16 Only a few authors focused on a specific pattern of BF; more often studies included various BF patterns.

The PROBIT study 25,26 was the only randomized trial, but the primary outcome of this cluster randomized trial was the effect of $\mathrm{BF}$ promotion on $\mathrm{BF}$ duration and exclusivity, and the study was not powered to detect the effects of BF duration or intensity on later overweight and obesity. 27

Childhood body mass index, overweight/obesity-Six systematic reviews examined the effects of BF on later BMI and risk of overweight and obesity9,17,19,20,21,22 (all 
reviews rated as of moderate quality) as did two systematic reviews of systematic reviews. 23,24 Despite important differences in the included reviews, such as the inclusion/exclusion criteria, they consistently reported a protective effect of BF on overweight and obesity (assessed during different periods of life, from early childhood up to adulthood, depending on the included studies).

The most recent 2015 meta-analysis by Horta et al.22 included 105 studies and confirmed that BF is associated with a reduced likelihood of obesity or overweight in childhood and adulthood (OR 0.74, 95\% CI: 0.70 to 0.78 ), with similar findings but a somewhat smaller effect size in analyses restricted to high quality studies (OR $0.87,95 \%$ CI: 0.76 to 0.99 ). This is in line with the results of previously published reviews9,17,19,20,21 and two systematic reviews of systematic reviews. 23,24

In summary, a consistent association of BF with a modest reduction by $13 \%$ of the odds of later overweight and obesity in childhood and adult life was reported, but residual confounding cannot be excluded.

Effect of the duration of exclusive breastfeeding-A 2012, high quality, Cochrane review, 18 which compared the effects of exclusive BF for six months with exclusive BF for three to four months (with subsequent mixed BF to six months), concluded that 'exclusive breastfeeding for six months does not seem to confer any long-term (at least to early school age) protection against obesity'. However, this conclusion was based solely on the findings of the underpowered PROBIT study.27

A 2013 review by Hornell et al.17 (moderate quality) included five studies summarizing data on exclusive $\mathrm{BF}$ and its association with the subsequent risk of overweight/obesity from one to six years of age. Three prospective cohort studies reported a lower risk of overweight or obesity with a longer duration of exclusive BF. In contrast, two studies, one the PROBIT study, found no consistent association between BF duration and exclusivity with regard to risk of overweight and obesity assessed at the ages of one, two, three, and 6.5 years.

A 2005 review by $O$ wen et al.19 (moderate quality) reported on three studies evaluating exclusive $\mathrm{BF}$ for eight months or more versus a shorter duration of BF, where the mean difference in mean BMI between groups, measured between two and 12 years of age, was $-0.39 \mathrm{~kg} \mathrm{~m}^{-2}$ (95\% CI: -0.51 to -0.26 ). However, this effect was no longer evident in two of the three included studies after adjustment for confounding factors such as socioeconomic status, maternal BMI, and maternal smoking in pregnancy (from -0.4 to -0.02 , after adjustment).

In summary, there is no conclusive evidence that exclusive BF, regardless of its duration, has a strong protective effect on the later risk of overweight and obesity.

Effect of the duration of any breastfeeding-Five systematic reviews9,16,17,20,21 assessed the later effect of the duration of any BF on BMI and/or the risk of overweight/ obesity; some were of moderate quality, apart from that by Harder et al.16 that was of low quality. 
The systematic review by Yan et al.21 (moderate quality) including 25 studies concluded that BF was associated with a reduced risk of obesity in children (adjusted OR $0.78 ; 95 \%$ CI: 0.74 to 0.81 ). Categorical analysis of 17 studies revealed a dose-response effect between $\mathrm{BF}$ duration and reduced risk of childhood obesity (any BF for seven and more months, adjusted OR $0.79,95 \%$ CI: 0.70 to 0.88 ). The quality of the included studies was not assessed by the authors, decreasing the reliability of the conclusions.

Likewise, Hornell et al.17 (moderate quality) reported a protective effect of duration of BF against later overweight and obesity in childhood, adolescence, and adulthood. The authors concluded that the evidence was convincing for this association with adolescent overweight/ obesity and limited, but suggestive for adulthood overweight/obesity.

Harder et al.16 (low quality) reported the results of a categorical analysis that confirmed a dose-response association between the duration of $\mathrm{BF}$ and risk of childhood and adulthood overweight based on BMI in the majority of the studies, assessed from 0 to 33 years (less than one month of BF, OR 1.0, 95\% CI: 0.65 to 1.55 ; one to three months of BF, OR 0.81, $95 \%$ CI: 0.74 to 0.88 ; four to six months of BF, OR $0.76,95 \%$ CI: 0.67 to 0.86 ; seven to nine months of BF, OR $0.67,95 \%$ CI: 0.55 to 0.82 ; more than nine months of BF, OR 0.68 , $95 \%$ CI: 0.50 to 0.91 ). One month of $\mathrm{BF}$ was associated with a four percent decrease in risk (OR 0.96 per month of BF, 95\% CI: 0.94 to 0.98 ). The quality of the included studies was not assessed.

Weng et al.20 (moderate quality) found conflicting evidence for duration of BF. Four of the five studies included in their review found no significant association between the duration of $\mathrm{BF}$ and risk of childhood overweight. However, the risk of bias differed greatly between the studies.

A 2005 review by Owen et al.19 (moderate quality) found that compared with BF for a shorter time, prolonged BF (not defined) provided a greater protective effect on mean levels of adiposity in children and adults from one to 70 years (based on BMI).

In summary, there are some indications that BF of very short duration has a lesser protective effect than $\mathrm{BF}$ of longer duration on the later risk of overweight and obesity, although residual confounding cannot be excluded.

Exclusive breastfeeding -With respect to BF exclusivity and its association with BMI when compared to any BF, Owen et al. reported that "the difference in mean BMI appeared somewhat smaller between 20 studies in which initial feeding groups were definitely exclusive (-0.06; 95\% CI: -0.09, -0.04) and 12 studies that did not report whether feeding was exclusive (-0.13; 95\% CI: -0.18, -0.08). However, this difference was not statistically significant $(P=0.45)$.'19

Included amongst the reviews was a 2012 systematic review and meta-analysis by Gale et al. 15 (moderate quality), which also investigated the contemporary (immediate) effect of BF or formula feeding on the body composition of healthy, term infants. The authors concluded that compared to $\mathrm{BF}$, formula feeding was associated with a transient lower fat mass and a higher fat-free mass from three to six months of age. 
In summary, there is no conclusive evidence that exclusive BF has a stronger protective effect on the later risk of overweight and obesity than predominant or partial BF.

\section{Infant Formula}

Eight systematic reviews assessed the effects of different components of infant formula feeding on BMI, overweight, obesity, or body composition (Table S2),

$28,29,30,31,32,33,34,35$ including two30,34 which addressed only the immediate consequences. All reviews included exclusively or mainly randomized controlled trials (RCTs).

Variations in terms of assessed components of infant formulas and selected outcomes were observed between the included reviews. Based on the AMSTAR assessment (Table S3), only one of the reviews was of high quality; 29 four reviews were classified as being of moderate quality,28,30,31,33 and two, of low quality.32,35

\section{- Protein content-Body composition}

Both a review by Patro-Gołą et al. 201629 (high quality) and a systematic review by Abrams et al. 201528 (moderate quality) identified data only on the immediate consequences of lowering the protein composition of infant formula feeding and concluded that the evidence was insufficient to determine whether reducing the protein content in infant formula influences infant body fat composition.

\section{Body mass index, overweight, obesity}

A systematic review by Patro-Gołą et al. 201629 identified four publications (two RCTs) that evaluated both immediate and later effects of different protein contents in infant formulae on BMI. No significant differences were found for mean BMI and BMI-z-score below six months of life. Starting from 12 months of age, one RCT (the CHOP Study) with a large sample size reported data on mean BMI and BMI-z-scores at later ages. BMI was significantly lower in the lower-protein formula group compared with the higher-protein formula group at all time points (i.e., at six, 12, and 24 months and at six years). Similarly, CHOP was the only study to report on the protein content of infant formula and the risk of becoming obese and found a markedly reduced obesity prevalence in the lower-protein formula group (1.77 and $2.2 \mathrm{~g}$ protein $100 \mathrm{kcal}^{-1}$ in infant and follow-on formulae, respectively) at the age of six compared with the higher-protein formula group ( 2.9 and $4.4 \mathrm{~g}$ protein $100 \mathrm{kcal}^{-1}$, respectively) (one RCT $n=448$, unadjusted Relative Risk (RR) $0.44,95 \%$ CI: 0.21 to 0.91 ; adjusted RR $0.35,95 \%$ CI: $0.15,0.82) .36$

A 2015 review by Abrams et al.28 also referred to the findings of the CHOP study with respect to BMI and obesity risk. Here, an additional RCT was included, reporting that the BMI at 24 months of age was lower in infants of overweight mothers fed lower-protein formula compared to controls. 37

In summary, lowering the protein content of infant formula is a promising intervention to reduce the risk of later overweight and obesity in children, but more studies replicating the effects on long-term health outcomes are needed. 


\section{- Addition of selected bacteria or non-digestible carbohydrates to infant formulae-Body composition and BMI}

No reviews relevant to longer-term childhood BMI or body composition effects were identified. A systematic review by Szajewska \& Chmielewska 201330 (moderate quality) investigated immediate effects of infant formula supplementation with different strains of bacteria, finding no significant difference in BMI between groups. Similarly, supplementation with inulin-type-fructans was summarized by Liber \& Szajewska34 (moderate quality), but no studies that assessed BMI were identified. None of the included trials reported on body composition.

In summary, there is no evidence to demonstrate long-term (or immediate) effects of infant formula supplemented with selected so-called "probiotic" bacteria or non-digestible carbohydrates on BMI.

\section{- Infant formula long-chain polyunsaturated fatty acids (LCPUFA) content- Body composition and BMI}

A 2012 systematic review by Campoy et al.31 (moderate quality) found no RCTs that assessed BMI. Body composition was not an outcome of interest in this systematic review.

A 2012 review by Rodriguez et al.32 (low quality) assessed the effects of n-3 LCPUFA intake, during pregnancy and postnatally, on infants' and young children's body composition. Six RCTS (four conducted in preterm and two, in term infants) focused on infant formulae supplemented with n-3 LCPUFA. The first of the two RCTs in term infants (classified as of moderate quality) showed, compared to an unsupplemented formula group, no effect of supplementation of infant formula with docosahexaenoic acid (DHA) or both DHA and arachidonic acid from birth to 17 weeks of age on body composition assessed at the age of 12 months. In the second RCT, in which the intervention was introduced from the first one to five days of life until the end of the second postnatal month, and the children were studied at 9 years of age, a similar lack of an effect on BMI and overweight risk in all of the study groups (LCPUFA-supplemented formula group, unsupplemented formula group, and breastfed group) was observed. However, the authors classified this study as being at high risk of bias. This was confirmed by the later review of Voortman et al.33 (moderate quality) evaluating the effects of PUFA dietary intake and blood levels during pregnancy, lactation, or early childhood on obesity and other cardio-metabolic outcomes.

In summary, there is no evidence that consumption of LCPUFA-supplemented infant formula reduces the risk of later obesity.

\section{- Soy-based infant formula-Body mass index}

A 2002 systematic review by Mendez et al. (low quality) assessed growth of infants fed soybased formula.35 Based on the results of one retrospective cohort study, there was no difference in BMI assessed in young adults who were participants of infant feeding trials and fed soy-based formula compared to cow's milk-based formula during infancy. 
In summary, there is no evidence that consumption of soy-based formula reduces the risk of later overweight or obesity.

\section{Complementary Feeding}

Five systematic reviews reported on various timings of complementary feeding (CF) introduction, 20,38,39,40,41 and one systematic review reported on the effects of types of foods introduced during the CF period, 42 both addressing immediate and later effects.

\section{- Timing of complementary feeding introduction-Body mass index and overweight, obesity}

A 2015 systematic review by Qasem et al.40 (moderate quality) aimed to summarize the influence of CF introduction at four months versus six months of age, but no significant differences between groups in BMI at six months of age were found.

A 2013 review by Pearce $\&$ colleagues39 (moderate quality) is an update of the 2011 systematic review by Moorcroft et al.38 (moderate quality) in which the majority of studies showed no clear association between the age of introduction of solid foods and obesity during infancy and childhood. This update applied different inclusion criteria (i.e., incorporating populations from developing countries, but also limiting the lower mean age of children when outcomes were assessed to four years of age). Of the 23 included, 21 studies assessed the relationship between the timing of CF and later childhood BMI (measured from two to 19 years of age). No significant association was found in 13 studies, and in another three studies, the observed effect was no longer significant when adjusted analysis was performed. However, it was noted that there is a possibility that very early introduction of solids may have an impact on BMI, based on results from two of four identified studies that assessed very early introduction of CF (at three and less than three months). The authors concluded that 'there is no clear association between age of introduction of complementary feeding and childhood overweight or obesity, but some evidence suggests that very early introduction (at or before 4 months), rather than at four to six months or more than six months, may increase the risk of childhood overweight' measured at four to 12 years of age.

A 2012 systematic review by Weng et al.20 (moderate quality) included prospective observational studies following up children from birth for at least two years to determine risk factors for childhood obesity. Among 30 included studies, four investigated the relationship between the earlier introduction of solid foods and childhood overweight at three (two studies), ten, and 11 years of age. There was some evidence supporting early introduction of solid foods as a risk factor for later overweight, but this was limited to formula-fed infants only. One of the two studies conducted in formula-fed infants found that compared with infants given solid foods after four months, infants given solid foods before four months were 1.12 times (95\% CI: 1.02 to 1.23 ) more likely to be overweight at three years of age. The second study found that, compared with infants who were given solid foods between four and five months of age, infants given solid foods before four months were 6.3 times (95\% CI: 2.3 to 16.9 ) more likely to be overweight at three years of age. However, this relationship was not significant in two studies of breastfed infants. A 2001 
review by Lanigan et al.41 (low quality) included no additional studies compared to those described above.

\section{Adiposity}

A review by Qasem et al. 201540 (moderate quality) found no difference in fat mass between groups that received $\mathrm{CF}$ at four months versus six months of age, when studied at six months of age. In contrast, an earlier review by Lanigan et al. 200141 (low quality) found in two studies an increased fat mass at follow-up in seven-year-old children who had $\mathrm{CF}$ introduced before 15 weeks compared with six-year-old children who had CF introduced after five months. However, two of the remaining systematic reviews (both of moderate quality) found no clear association between the timing of CF introduction and measures of adiposity such as skinfold thicknesses, fat mass, and percentage fat mass measured by DXA and/or bioelectrical impedance.38,39

In conclusion, there is no consistent evidence of effects of the timing of introducing $\mathrm{CF}$ and later adiposity risk.

In summary, there is no consistent evidence of an association between the earlier introduction of CF before 15 weeks/four months and later childhood adiposity.

\section{- Types of complementary food-BMI, childhood obesity}

Only one systematic review by Pearce \& Langley-Evans 201342 (moderate quality) analyzed whether the risk of subsequent overweight or obesity was affected by the type of $\mathrm{CF}$ introduced during infancy. Ten observational studies were included. The authors assessed different types of foods/food groups and found no association between the types of food given and childhood obesity in three out of four studies. One study found that delaying the age at which introduction of solid and semi-solid foods were introduced (bread and biscuits mixed with milk) had an inverse association with the BMI z-score at ten and 11 years of age, after adjustments. One of the included studies analyzed the association between adherence to a specific diet during infancy and BMI at four years of age. Neither fat mass nor BMI changes were associated with $\mathrm{CF}$ of 'a diet based on fruit, vegetables, meat, fish and homeprepared foods'. Evaluation of specific macronutrient's effects in regard to the CF period, together with quality assessment of the included studies, is presented elsewhere in this review.

In summary, there is no evidence to suggest associations between certain CF types or patterns and subsequent childhood overweight or obesity.

\section{Protein Intake in Early Childhood}

Pearce \& Langley-Evans 201342 (moderate quality) also investigated the effects of protein (total protein content or type of protein) consumed during the CF period by infants on the risk of overweight or obesity and body composition in childhood. Based on conflicting data from four cohort studies, the authors concluded that "Some association was found between high protein intakes at 2-12 months of age and higher body mass index (BMI) or body fatness in childhood, but was not the case in all studies." 
Hornell et al. 201343 (moderate quality) assessed the effects of different levels of protein intake (and different sources of protein) during infancy and childhood on different health outcomes, including BMI and body composition, in a Nordic setting. For the purpose of a subanalysis limited to the effects of early protein intake, the authors included 13 studies (two clinical trials, nine cohort studies, and two cross-sectional studies) in which the association of protein intake in children from 0 to approximately 3 years of age (only in 2 studies the age range was from 1 to 5 years) with BMI, overweight /obesity, or body composition (up to ten years of age) was evaluated. They concluded that higher protein intake during the first years of life is positively associated with increased growth and higher BMI later in life. The evidence was classified by the authors as convincing (grade one), when taking into account the results of the studies and evidence quality assessment. It needs to be emphasized, however, that this review incorporated data on the effects of protein intake and protein content in infant formula but used these terms synonymously. The evidence (data from four cohort studies and one cross-sectional study) was described by the authors as "limitedinconclusive (grade 4)" considering the conflicting results of the identified studies with respect to the effects of protein intake in early childhood on later body fat.

These two reviews varied greatly in terms of applied search strategy and eligibility criteria, which may explain differences in study selection and the important discrepancy in obtained results regarding BMI and obesity risk.

In summary, there is inconsistent evidence of an association between a higher dietary protein intake in early childhood beyond infancy and later childhood overweight or obesity.

\section{Fat Intake in Early Childhood}

\section{- Total fat intake-BMI and body composition}

A 2014 Cochrane review (high quality) assessed the effects of fat intake in infancy on childhood outcomes of interest.44 Four observational studies conducted in children aged up to three years were included. In one cohort (two analyses), the authors addressed associations of dietary fat intake at different time points (including second and third year of life) with percentage body fat at 70 months and with BMI at eight years of age. A direct relationship of fat intake with BMI or BMI change, as well as with the percentage of body fat, was noted. However, analyses of the other studies found no association, or even an inverse association to that expected. In one, greater total fat intake at two years was related to a lower percentage of subscapular skinfold and fat mass after 18 years of follow-up (but not BMI or other skinfolds). The authors concluded that there was no clear association between lower total fat intake and later BMI and/or body composition.

In summary, there is no conclusive evidence of a relationship between fat intake in the first years of life and childhood BMI or early adulthood adiposity.

\section{- Polyunsaturated fatty acid intake-BMI, body composition, and obesity}

A 2015 review by Voortman et al.33 (moderate quality) assessed the influence of the intake and status of LCPUFA in infancy on later obesity (including effects on BMI and body fat). 
Eight interventional and observational studies conducted during early childhood that assessed different outcomes of interest were included. Three of the included interventional studies were performed in term infants and assessed different forms of PUFA intake (fish oils, dietary supplements), with different comparisons (placebo, other fats). Two reported no difference in BMI or body fat percentage immediately after the intervention, and another showed no long-term effect of the intervention on BMI at eight years (interventions starting from six months to five years). Five of the included cohort studies evaluated the intake or levels of PUFA at various time-points (from birth until five years of age). Long-term effects were assessed after one to ten years of follow-up and showed conflicting evidence. In three studies, no consistent effects were noted. In one study, a lower obesity risk at four years was found (dietary intake of PUFA evaluated at 12 to 19 months). In contrast, in another study, a higher BMI was found at ten years of age in a sub-group with higher n-3 and lower n- 6 fatty acids levels at birth (in cord blood). The authors concluded that there was insufficient evidence to suggest an effect of polyunsaturated fatty acid intake in early childhood on later overweight or obesity. A 2014 review by Koletzko et al.45 (low quality) included no additional studies compared to those described above.

In summary, there is no conclusive evidence that PUFA intake in early childhood influences long-term risk of overweight, obesity, or body fat content.

\section{Sugars and Sugar-Sweetened Beverages}

A total of seven systematic reviews $46,47,48,49,50,51,52$ and one systematic review of systematic reviews53 evaluated later as well as more immediate effects of sugar-sweetened beverages (SSB) and dietary sugar intake on childhood overweight and obesity. In the age group up to three years, a single RCT and different types of observational studies were included. The majority of the reviews were of low quality,46,47,48,49,50 and two, of high quality.51,52 Two reviews48,51 (including one of high quality) 51 included our population of interest in the inclusion criteria; however, none identified any studies of exposures in children aged up to three years, and they are not described in detail.

Childhood BMI-In a 2013 review by Perez-Morales et al.47 (low quality), three out of seven longitudinal studies evaluating influences of exposure up to the age of three years focused on SSB intake. The authors concluded that there is 'a trend showing that high consumption of SSB is associated to higher BMI, waist circumference, and overweight later in childhood'. In only one study (out of three related to our focus of interest), regular consumers of SSB at two years compared with 'non-consumers' had a higher risk of overweight at 4.5 years (OR 2.4; 95\%CI: 1.11 to 5.05) independent of the overall nutritional content of the diet.54 In another study, no statistically significant association was noted between consumption of sweet drinks at the age of two to three years and development of overweight one year later among children who were non-overweight at baseline, however, a significant difference was found in children who were already at risk for overweight at baseline (BMI $85^{\text {th }}$ to $<95^{\text {th }}$ percentile). 55 Similarly, increases in added sugar intake from SSB between the ages of one and two years were not associated with later BMI at the age of seven years in another study.56 
In a systematic review by Osei-Assibey et al. 201249 (low quality), only two studies conducted in children aged up to three years were identified. One RCT, focusing on the high risk for obesity in a population of American Indians/Alaskan Natives,57 showed that combined community and family interventions to promote breastfeeding (at visits before birth) and a reduction in the consumption of SSB (at visits within the first three months), rather than a community-alone intervention, decreased the BMI z-score rise in toddlers at the age of two years. Among longitudinal studies, only one was conducted in children aged two to three years, reported above, 55 which was also included in other systematic reviews.46, 50

Childhood overweight/obesity-A systematic review and meta-analysis by Te Morenga et al. 201252 (high quality) evaluated the influence of dietary sugar intake on childhood overweight and obesity after one year of follow-up. Among the 21 included studies, six reported children who were recruited under the age of three years. The authors found an association between one daily serving intake of SSB at baseline (versus children consuming none or very little) and odds of being overweight at follow-up (OR 1.55; 95\%CI: 1.32 to 1.82). However, this result should be treated with caution, as different populations/ comparisons (including adolescents, obese children) were included and there may be effects of lasting dietary patterns rather than of early programming. Similar findings were reported in a systematic review of systematic reviews by Keller et al..53

Additionally, in a systematic review by Gibson46 (low quality), one cross-sectional study showed a positive association between soda consumption and overweight amongst children who were two years of age.

In summary, there is inconsistent evidence to suggest an association between SSB intake in early childhood and long-term overweight and obesity; current diet is likely to be a major confounder.

Pearce \& Langley-Evans 201342 reported one cohort study that focused on energy intake at four months of age and its influence on BMI; this study found a linear association between energy intake and higher BMI at one, two, three, and five years of age in formula-fed or mixed-fed infants only. Each $420 \mathrm{~kJ}$ per day increase in energy at four months led to a increase in risk of BMI > 85th percentile with borderline significance (OR 1.25; 95\% CI: 1.00 to 1.55$)$. Higher energy intake at four months was also positively associated with rapid weight gain, which independently predicted the risk of obesity in children. 42 The quality of the identified study, assessed with the use of Newcastle-Ottawa scale, was quite high, although no adjustment for dietary intake at time of measurement (age one, two, three, and five years) was reported. Based on these findings, the authors stated that "Higher energy intake during complementary feeding was associated with higher BMI in childhood". However, they also emphasized that considering the limited body of evidence (one single study), the findings were inconclusive.

In summary, there is limited evidence that total energy intake in early childhood is associated with later higher BMI. However, confirmation of this association by controlled intervention studies is not available. 


\section{Dairy Food Intake}

No reviews were found that addressed the effect of exposure to milk or dairy products in early life on later BMI or body composition in children other than the review by Pearce \& Langley-Evans 42 which concluded, when referring to long-term consequences of different protein intakes in infancy, that "particularly dairy protein, in infancy could be associated with an increase in BMI and body fatness, but further research is needed to establish the nature of the relationship." In relation to immediate effects, a recent review by Dror et al.58 (moderate quality) found no significant association between total dairy or total milk intake and BMI z-score at two years or incident overweight at three years, in line with a previous 2011 review on the relationship between dairy consumption and overweight/obesity in children and adults.59

In summary, there is inconsistent evidence of an association of dairy consumption in early childhood with increased later overweight and obesity, but there is a lack of relevant controlled intervention studies.

\section{Fruit and Vegetable Intake}

No reviews were found that addressed associations between dietary intake of fruits and vegetables in early childhood on later body composition, overweight, and obesity.

In a review by Kaiser et al. 201460 (moderate quality) that aimed to summarize the evidence on 'the effectiveness of the general recommendation to eat more fruits and vegetables for weight loss or the prevention of weight gain', no studies in children were identified.

In summary, there is no evidence that the dietary intake of fruits and vegetables in early childhood affects later body composition, overweight, and obesity.

\section{Adherence to Dietary Guidelines}

The influence of adherence to dietary guidelines was also addressed by Pearce $\&$ LangleyEvans.42 Based on the results of one identified study, no association was found between the Infant Guideline Score (IGS, based on dietary patterns) and later childhood BMI, fat mass, or fat-mass index. Positive associations between increasing IGS and both lean mass and the lean mass index were observed in four-year-old children. The overall quality of this study was moderate, and no firm conclusions can be drawn from a single report.

In summary, there is insufficient evidence of any effects of adherence to dietary guidelines in early childhood on later body composition, overweight, and obesity.

\section{Discussion}

\section{Summary of main findings}

The aim of this systematic review of systematic reviews was to examine existing evidence on different nutritional interventions or exposures in infants and children and their effects on later health, specifically outcomes related to overweight and obesity. We wished to address the 'early life programming' hypothesis, which suggests that there are periods of developmental vulnerability to nutritional exposures when suboptimal nutrition may lead to 
persistent phenotypic changes in later life and increased risk of obesity.6,7 To achieve this goal, in reviewing the literature we attempted to distinguish immediate effects from later effects of early nutrition. However, there is no clearly defined cut-off time point to indicate the optimal time for assessment of the phenotypic effect of programming either in relation to the interval following the exposure or the age of the offspring. Moreover, many of the selected reviews included studies on both immediate and longer-term effects of the nutritional exposures, making conclusions sometimes difficult to be formulated. Our approach was to identify the age of participants during the intervention and outcome assessment, while attempting to arbitrarily classify observed effects as immediate or later. On this basis, we found no strong evidence that any of the nutritional interventions or exposures in children aged up to three years consistently reduces the risk of overweight/ obesity or increased adiposity. There was, however, weak evidence that some of these interventions/exposures may be associated with a reduced risk of overweight/obesity. A consistent association of breastfeeding with a modest reduction in the risk of later overweight and obesity in childhood and adulthood was found (the odds decreased by $13 \%$ based on high-quality studies), but residual confounding cannot be excluded. Lowering the protein content of infant formula is a promising intervention to reduce the risk of later overweight and obesity in children. There is no consistent evidence of an association of the age of introducing complementary foods, sugar-sweetened beverage, or energy intake in early childhood, with later overweight/obesity, but there are some indications of an association of protein intake during the complementary feeding period with later overweight/obesity.

\section{Strengths and limitations of this review}

This review summarizes evidence from a number of systematic reviews of different nutritional interventions for the same problem, thus, it differs from systematic reviews that summarize original studies. Our search strategy was developed to ensure a thorough literature search, with no restrictions by language. All analyses were defined a priori. Considering that for some interventions (e.g., breastfeeding), RCTs would have been unethical, systematic reviews of non-RCTs were included. However, our review might not be comprehensive. Moreover, reviews were included only if our population and outcomes of interest were clearly identified in the abstract. If not, these systematic reviews might have been omitted.

The majority of the included reviews were published between 2010 and 2015. The inclusion of older systematic reviews, which have not been updated, may be challenged. However, for completeness, we reviewed all available systematic reviews, as small differences in search strategies in the included reviews might have resulted in substantial differences in the set of studies identified. As a consequence, often the evidence from included reviews is overlapping. Nonetheless, whenever possible, we aimed to base our conclusions on the most recent and robust reviews to avoid overestimating the effect.

Only some of the reviews included in our review were of high methodological quality based on the AMSTAR assessment. We acknowledge that originally this tool was developed to assess the reviews of RCTs only61 and that other tools may be considered (including the 
newly developed ROBIS tool).62 However, all of the assessed elements apply also to systematic reviews of observational studies, and this tool had been previously used by different authors of reviews of observational studies.24,53

The conclusions of the original systematic reviews were often limited by the small number of included trials and/or participants and/or by the heterogeneity of the studies included. Except for the Cochrane reviews, which consistently used the Cochrane Risk of Bias tool, in the other original systematic reviews, the risk of bias in the included studies was not always assessed. When evaluated, often the included studies were not methodologically rigorous.

\section{Conclusions}

Rigorous testing of the 'early life programming' hypothesis requires clarity on both the timing of an exposure and outcome assessment in order to distinguish immediate consequences of dietary interventions from longer-term and potentially programmed effects. More high quality, controlled interventional studies are needed to address specific relationships between some nutritional components in early life and later risk of adiposity, ranging from protein, fat, and SSB intake to overall diet quality as well as the consumption of different type of foods such as dairy products or fruits and vegetables. In addition, further research is needed to identify the most effective strategies to implement particular dietary recommendations.

\section{Supplementary Material}

Refer to Web version on PubMed Central for supplementary material.

\section{Acknowledgements}

The research leading to these results has received funding from the European Union's Seventh Framework Programme (FP7/2007- 2013), project EarlyNutrition under grant agreement No. 289346.

KMG was supported by the National Institute for Health Research through the NIHR Southampton Biomedical Research Centre and by the European Union's Seventh Framework Programme (FP7/2007-2013), projects EarlyNutrition and ODIN under grant agreement numbers 289346 and 613977.

LP was supported in the UK by Tommy's Charity, the National Institute for Health Research through the NIHR Biomedical Research Centre at King's College London and Guys and St. Thomas' NHS Foundation Trust and by the European Union's Seventh Framework Programme (FP7/2007-2013) project EarlyNutrition under grant agreement No. 289346.

\section{Abbreviations}

$\begin{array}{ll}\text { RCT } & \text { randomized controlled trial } \\ \text { BMI } & \text { body mass index } \\ \text { BF } & \text { breastfeeding } \\ \text { CF } & \text { complementary feeding } \\ \text { CHOP } & \text { Childhood Obesity Program }\end{array}$




\section{References}

1. Barker DJ. The developmental origins of adult disease. Eur J Epidemiol. 2003; 18:733-736. [PubMed: 12974544]

2. Widdowson EM, McCance RA. The effect of finite periods of undernutrition at different ages on the composition and subsequent development of the rat. Proc R Soc Lond B Biol Sci. 1963; 158:32942. [PubMed: 14070049]

3. Williams TC, Drake AJ. What a general paediatrician needs to know about early life programming. Arch Dis Child. 2015; 100:1058-1063. [PubMed: 25990501]

4. Singhal A, Lucas A. Early origins of cardiovascular disease: is there a unifying hypothesis? Lancet. 2004; 363:1642-5. [PubMed: 15145640]

5. Baird J, Fisher D, Lucas P, Kleijnen J, Roberts H, Law C. Being big or growing fast: systematic review of size and growth in infancy and later obesity. BMJ. 2005; 331:929. [PubMed: 16227306]

6. Koletzko B, Chourdakis M, Grote H, et al. Regulation of early human growth: impact on long-term health. Ann Nutr Metab. 2014; 65:101-109. [PubMed: 25413647]

7. Koletzko B, Brands B, Chourdakis M, et al. The Power of Programming and The Early Nutrition Project: opportunities for health promotion by nutrition during the first thousand days of life and beyond. Ann Nutr Metab. 2014; 64:187-196.

8. Koletzko B, von Kries R, Closa R, et al. European Childhood Obesity Trial Study Group. Lower protein in infant formula is associated with lower weight up to age $2 \mathrm{y}$ : a randomized clinical trial. Am J Clin Nutr. 2009; 89:1836-1845. [PubMed: 19386747]

9. Arenz S, Rückerl R, Koletzko B, von Kries R. Breast-feeding and childhood obesity--a systematic review. Int J Obes Relat Metab Disord. 2004; 28:1247-1256. [PubMed: 15314625]

10. Commission on Ending Childhood Obesity. Report of the Commission on Ending Childhood Obesity. World Health Organization; Geneva: 2016. [WWW document] URL http://www.who.int/ end-childhood-obesity/en/ [accessed June 5, 2016]

11. Zalewski B, Patro B, Veldhorst M, et al. Nutrition of infants and young children (1-3 years) and its effect on later health: A systematic review of current recommendations (EarlyNutritrion Project). Crit Rev Food Sci Nutr. 2015 Mar. 9:0. [Epub ahead of print].

12. Higgins, JPT., Green, S., editors. Cochrane Handbook for Systematic Reviews of Interventions Version 5.1.0. The Cochrane Collaboration; 2011. Available from www.cochrane-handbook.org [updated March 2011]

13. Smith V, Devane D, Begley CM, Clarke M. Methodology in conducting a systematic review of systematic reviews of healthcare interventions. BMC Med Res Methodol. 2011; 11:15. [PubMed: 21291558]

14. Shea BJ, Hamel C, Wells GA, et al. AMSTAR is a reliable and valid measurement tool to assess the methodological quality of systematic reviews. J Clin Epidemiol. 2009; 62:1013-1020. [PubMed: 19230606]

15. Gale C, Logan KM, Santhakumaran S, Parkinson JRC, Hyde MJ, Modi N. Effect of breastfeeding compared with formula feeding on infant body composition: A systematic review and metaanalysis. Am J Clin Nutr. 2012; 95:656-669. [PubMed: 22301930]

16. Harder T, Bergmann R, Kallischnigg G, Plagemann A. Duration of breastfeeding and risk of overweight: a meta-analysis. Am J Epidemiol. 2005; 162:397-403. [PubMed: 16076830]

17. Hornell A, Lagstrom H, Lande B, Thorsdottir I. Breastfeeding, introduction of other foods and effects on health: a systematic literature review for the 5th Nordic Nutrition Recommendations. Food Nutr Res. 2013:57.

18. Kramer MS, Kakuma R. Optimal duration of exclusive breastfeeding. Cochrane Database Syst Rev. 2012; 8:CD003517.

19. Owen CG, Martin RM, Whincup PH, Davey-Smith G, Gillman MW, Cook DG. The effect of breastfeeding on mean body mass index throughout life: a quantitative review of published and unpublished observational evidence. Am J Clin Nutr. 2005; 82:1298-1307. [PubMed: 16332664]

20. Weng SF, Redsell SA, Swift JA, Yang M, Glazebrook CP. Systematic review and meta-analyses of risk factors for childhood overweight identifiable during infancy. Arch Dis Child. 2012; 97:10191026. [PubMed: 23109090] 
21. Yan J, Liu L, Zhu Y, Huang G, Wang PP. The association between breastfeeding and childhood obesity: a meta-analysis. BMC Public Health. 2014; 14:1267. [PubMed: 25495402]

22. Horta BL, Loret de Mola C, Victora CG. Long-term consequences of breastfeeding on cholesterol, obesity, systolic blood pressure and type 2 diabetes: a systematic review and meta-analysis. Acta Paediatr Suppl. 2015; 104:30-37.

23. Ip S, Chung M, Raman G, Trikalinos TA, Lau J. A summary of the Agency for Healthcare Research and Quality's evidence report on breastfeeding in developed countries. Breastfeed Med. 2009; 1:S17-30.

24. Monasta L, Batty GD, Cattaneo A, et al. Early-life determinants of overweight and obesity: a review of systematic reviews. Obes Rev. 2010; 11:695-708. [PubMed: 20331509]

25. Kramer MS, Matush L, Vanilovich I, et al. A randomized breast-feeding promotion intervention did not reduce child obesity in Belarus. J Nutr. 2009; 139:417S-421S. [PubMed: 19106322]

26. Kramer MS, Matush L, Vanilovich I, et al. Effects of prolonged and exclusive breastfeeding on child height, weight, adiposity, and blood pressure at age $6.5 \mathrm{y}$ : evidence from a large randomized trial. Am J Clin Nutr. 2007; 86:1717-1721. [PubMed: 18065591]

27. Rückinger $S$, von Kries R. Breastfeeding and reduced risk of childhood obesity: will randomized trials on breastfeeding promotion give the definite answer? Am J Clin Nutr. 2009; 89:653-655. [PubMed: 19116325]

28. Abrams SA, Hawthorne KM, Pammi M. A systematic review of controlled trials of lower-protein or energy-containing infant formulas for use by healthy full-term infants. Adv Nutr. 2015; 6:178188. [PubMed: 25770256]

29. Patro-Golab B, Zalewski B, Kouwenhoven S, et al. Protein concentration in milk formula, growth, and later risk of obesity: A systematic review. J Nutr. 2016; 146:551-564. [PubMed: 26865649]

30. Szajewska H, Chmielewska A. Growth of infants fed formula supplemented with Bifidobacterium lactis Bb12 or Lactobacillus GG: a systematic review of randomized controlled trials. BMC Pediatr. 2013; 13:185. [PubMed: 24215626]

31. Campoy C, Escolano-Margarit MV, Anjos T, Szajewska H, Uauy R. Omega 3 fatty acids on child growth, visual acuity and neurodevelopment. Br J Nutr. 2012; 107:S85-106. [PubMed: 22591907]

32. Rodríguez G, Iglesia I, Bel-Serrat S, Moreno LA. Effect of n-3 long chain polyunsaturated fatty acids during the perinatal period on later body composition. Br J Nutr. 2012; 107:S117-128. [PubMed: 22591886]

33. Voortman T, Van Den Hooven EH, Braun KVE, et al. Effects of polyunsaturated fatty acid intake and status during pregnancy, lactation, and early childhood on cardiometabolic health: A systematic review. Prog Lipid Res. 2015; 59:67-87. [PubMed: 26025302]

34. Liber A, Szajewska H. Effects of inulin-type fructans on appetite, energy intake, and body weight in children and adults: systematic review of randomized controlled trials. Ann Nutr Metab. 2013; 63:42-54.

35. Mendez MA, Anthony MS, Arab L. Soy-based formulae and infant growth and development: a review. J Nutr. 2002; 132:2127-2130. [PubMed: 12163650]

36. Weber M, Grote V, Closa-Monasterolo R, et al. for The European Childhood Obesity Trial Study Group. Lower protein in infant formula reduces BMI and obesity risk at school age: follow-up of a randomized trial. Am J Clin Nutr. 2014; 99:1041-1051. [PubMed: 24622805]

37. Inostroza J, Haschke F, Steenhout P, Grathwohl D, Nelson SE, Ziegler EE. Low-protein formula slows weight gain in infants of overweight mothers: a randomized trial. J Pediatr Gastroenterol Nutr. 2014; 59:70-77. [PubMed: 24637965]

38. Moorcroft KE, Marshall JL, McCormick FM. Association between timing of introducing solid foods and obesity in infancy and childhood: a systematic review. Matern Child Nutr. 2011; 7:3-26. [PubMed: 21143583]

39. Pearce J, Taylor MA, Langley-Evans SC. Timing of the introduction of complementary feeding and risk of childhood obesity: a systematic review. Int J Obes (Lond). 2013; 37:1295-1306. [PubMed: 23736360]

40. Qasem W, Fenton T, Friel J. Age of introduction of first complementary feeding for infants: a systematic review. BMC Pediatr. 2015; 15:107. [PubMed: 26328549] 
41. Lanigan JA, Bishop J, Kimber AC, Morgan J. Systematic review concerning the age of introduction of complementary foods to the healthy full-term infant. Eur J Clin Nutr. 2001; 55:309-320. [PubMed: 11378803]

42. Pearce J, Langley-Evans SC. The types of food introduced during complementary feeding and risk of childhood obesity: a systematic review. Int J Obes (Lond). 2013; 37:477-485. [PubMed: 23399778]

43. Hörnell A, Lagström H, Lande B, Thorsdottir I. Protein intake from 0 to 18 years of age and its relation to health: a systematic literature review for the 5th Nordic Nutrition Recommendations. Food Nutr Res. 2013; 57

44. Hooper L, Abdelhamid A, Bunn D, Brown T, Summerbell CD, Skeaff CM. Effects of total fat intake on body weight. Cochrane Database Syst Rev. 2015; 8:CD011834.

45. Koletzko B, Boey CCM, Campoy C, et al. Current information and Asian perspectives on longchain polyunsaturated fatty acids in pregnancy, lactation and infancy. Systematic review and practice recommendations from an Early Nutrition Academy workshop. Ann Nutr Metab. 2014; 65:49-80. [PubMed: 25227906]

46. Gibson S. Sugar-sweetened soft drinks and obesity: a systematic review of the evidence from observational studies and interventions. Nutr Res Rev. 2008; 21:134-147. [PubMed: 19087367]

47. Pérez-Morales E, Bacardí-Gascón M, Jiménez-Cruz A. Sugar-sweetened beverage intake before 6 years of age and weight or BMI status among older children; systematic review of prospective studies. Nutr Hosp. 2013; 28:47-51.

48. Clabaugh K, Neuberger GB. Research evidence for reducing sugar sweetened beverages in children. Issues Compr Pediatr Nurs. 2011; 34:119-30. [PubMed: 21767071]

49. Osei-Assibey G, Dick S, Macdiarmid J, et al. The influence of the food environment on overweight and obesity in young children: a systematic review. BMJ Open. 2012; 2

50. Olsen NJ, Heitmann BL. Intake of calorically sweetened beverages and obesity. Obes Rev. 2009; 10:68-75. [PubMed: 18764885]

51. Kaiser KA, Shikany JM, Keating KD, Allison DB. Will reducing sugar-sweetened beverage consumption reduce obesity? Evidence supporting conjecture is strong, but evidence when testing effect is weak. Obes Rev. 2013; 14:620-633. [PubMed: 23742715]

52. Te Morenga L, Mallard S, Mann J. Dietary sugars and body weight: systematic review and metaanalyses of randomised controlled trials and cohort studies. BMJ. 2012; 346:e7492. [PubMed: 23321486]

53. Keller A, Bucher Della Torre S. Sugar-Sweetened Beverages and Obesity among Children and Adolescents: A Review of Systematic Literature Reviews. Child Obes. 2015; 11:338-346. [PubMed: 26258560]

54. Dubois L, Farmer A, Girard M, Peterson K. Regular sugar-sweetened beverage consumption between meals increases risk of overweight among preschool-aged children. J Am Diet Assoc. 2007; 107:924-934. discussion 934-935. [PubMed: 17524711]

55. Welsh JA, Cogswell ME, Rogers S, Rockett H, Mei Z, Grummer-Strawn LM. Overweight among low-income preschool children associated with the consumption of sweet drinks: Missouri, 1999-2002. Pediatrics. 2005; 115:223-229.

56. Herbst A, Diethelm K, Cheng G, Alexy U, Icks A, Buyken AE. Direction of associations between added sugar intake in early childhood and body mass index at age 7 years may depend on intake levels. J Nutr. 2011; 141:1348-1354. [PubMed: 21562234]

57. Karanja N, Lutz T, Ritenbaugh C, et al. The TOTS community intervention to prevent overweight in American Indian toddlers beginning at birth: a feasibility and efficacy study. J Community Health. 2010; 35:667-675. [PubMed: 20508978]

58. Dror DK. Dairy consumption and pre-school, school-age and adolescent obesity in developed countries: a systematic review and meta-analysis. Obes Rev. 2014; 15:516-527. [PubMed: 24655317]

59. Louie JC, Flood VM, Hector DJ, Rangan AM, Gill TP. Dairy consumption and overweight and obesity: a systematic review of prospective cohort studies. Obes Rev. 2011; 12:582-592. 
60. Kaiser KA, Brown AW, Bohan Brown MM, Shikany JM, Mattes RD, Allison DB. Increased fruit and vegetable intake has no discernible effect on weight loss: a systematic review and metaanalysis. Am J Clin Nutr. 2014; 100:567-576. [PubMed: 24965308]

61. Smith V, Devane D, Begley CM, Clarke M. Methodology in conducting a systematic review of systematic reviews of healthcare interventions. BMC Med Res Methodol. 2011; 11:15. [PubMed: 21291558]

62. Whiting P, Savović J, Higgins JP, et al. ROBIS group. ROBIS: A new tool to assess risk of bias in systematic reviews was developed. J Clin Epidemiol. 2016; 69:225-234. [PubMed: 26092286] 